\title{
Decoherence in supernova neutrino transformations suppressed by deleptonization
}

\author{
Andreu Esteban-Pretel,,${ }^{1}$ Sergio Pastor,${ }^{1}$ Ricard Tomàs, ${ }^{1}$ Georg G. Raffelt, ${ }^{2}$ and Günter Sigl ${ }^{3,4}$ \\ ${ }^{1}$ AHEP Group, Institut de Física Corpuscular, CSIC/Universitat de València, \\ Edifici Instituts d'Investigació, Apt. 22085, 46071 València, Spain \\ ${ }^{2}$ Max-Planck-Institut für Physik (Werner-Heisenberg-Institut), Föhringer Ring 6, 80805 München, Germany \\ ${ }^{3}$ II. Institut für theoretische Physik, Universität Hamburg \\ Luruper Chaussee 149, D-22761 Hamburg, Germany \\ ${ }^{4} \mathrm{APC}^{*}$ (AstroParticules et Cosmologie), 10, rue Alice Domon et Léonie Duquet, 75205 Paris Cedex 13, France
}

(Dated: 28 December 2007)

\begin{abstract}
In the dense-neutrino region at $50-400 \mathrm{~km}$ above the neutrino sphere in a supernova, neutrinoneutrino interactions cause large flavor transformations. We study when the multi-angle nature of the neutrino trajectories leads to flavor decoherence between different angular modes. We consider a two-flavor mixing scenario between $\nu_{e}$ and another flavor $\nu_{x}$ and assume the usual hierarchy $F_{\nu_{e}}>F_{\bar{\nu}_{e}}>F_{\nu_{x}}=F_{\bar{\nu}_{x}}$ for the number fluxes. We define $\epsilon=\left(F_{\nu_{e}}-F_{\bar{\nu}_{e}}\right) /\left(F_{\bar{\nu}_{e}}-F_{\bar{\nu}_{x}}\right)$ as a measure for the deleptonization flux which is the one crucial parameter. The transition between the quasi single-angle behavior and multi-angle decoherence is abrupt as a function of $\epsilon$. For typical choices of other parameters, multi-angle decoherence is suppressed for $\epsilon \gtrsim 0.3$, but a much smaller asymmetry suffices if the neutrino mass hierarchy is normal and the mixing angle small. The critical $\epsilon$ depends logarithmically on the neutrino luminosity. In a realistic supernova scenario, the deleptonization flux is probably enough to suppress multi-angle decoherence.
\end{abstract}

PACS numbers: 14.60.Pq, 97.60.Bw

\section{INTRODUCTION}

In the dense neutrino flux emerging from a supernova (SN) core, neutrino-neutrino refraction causes nonlinear flavor oscillation phenomena that are unlike anything produced by ordinary matter $[1,2,3,4,5,6,7,8,9$, 10, 11]. The crucial phenomenon is a collective mode of pair transformations of the form $\nu_{e} \bar{\nu}_{e} \rightarrow \nu_{x} \bar{\nu}_{x}$ where $x$ represents some suitable superposition of $\nu_{\mu}$ and $\nu_{\tau}$. This pair-wise form of flavor transformation leaves the net flavor-lepton number flux unchanged. Even an extremely small mixing angle is enough to trigger this effect that is insensitive to the presence of ordinary matter unless there is a Mikheyev-Smirnov-Wolfenstein (MSW) resonance in the dense-neutrino region.

Collective pair transformations require a large neutrino density and a pair excess of a given flavor. In typical SN models one finds a hierarchy of number fluxes $F_{\nu_{e}}>F_{\bar{\nu}_{e}}>F_{\nu_{x}}=F_{\bar{\nu}_{x}}$. The first part of the hierarchy is caused by the deleptonization of the collapsed core whereas the second is caused by the absence of chargedcurrent interactions for neutrino species other than $\nu_{e}$ and $\bar{\nu}_{e}$. The neutrino fluxes streaming from a collapsed star thus provide a natural environment for a flavor pair excess. On the other hand, the SN core itself is characterized by a large $\nu_{e}$ chemical potential that enhances the $\nu_{e}$ density and suppresses that of $\bar{\nu}_{e}$ so that here pair transformations cannot occur. Likewise, the deleptonization burst immediately after core bounce has an excess of $\nu_{e}$

*UMR 7164 (CNRS, Université Paris 7, CEA, Observatoire de Paris) and a depletion of $\bar{\nu}_{e}$ 12], suggesting that it is unaffected by collective pair transformations.

We illustrate collective pair conversions with a simple example in Fig. 1. assuming a typical SN neutrino luminosity to be quantified later. We show the evolution of the $z$-components of the global flavor polarization vector $\mathbf{P}$ for neutrinos and $\overline{\mathbf{P}}$ for antineutrinos, where initially $P=|\mathbf{P}|=1+\epsilon$ with $\epsilon=0.25$ and $\bar{P}=|\overline{\mathbf{P}}|=1$. We have assumed a monochromatic spectrum, that all neutrinos are emitted at $45^{\circ}$ relative to the radial direction, the atmospheric $\Delta m^{2}$, a small vacuum mixing angle $\sin 2 \theta=10^{-3}$ to mimic the effect of ordinary matter. and an inverted mass hierarchy. For the normal hierarchy, no

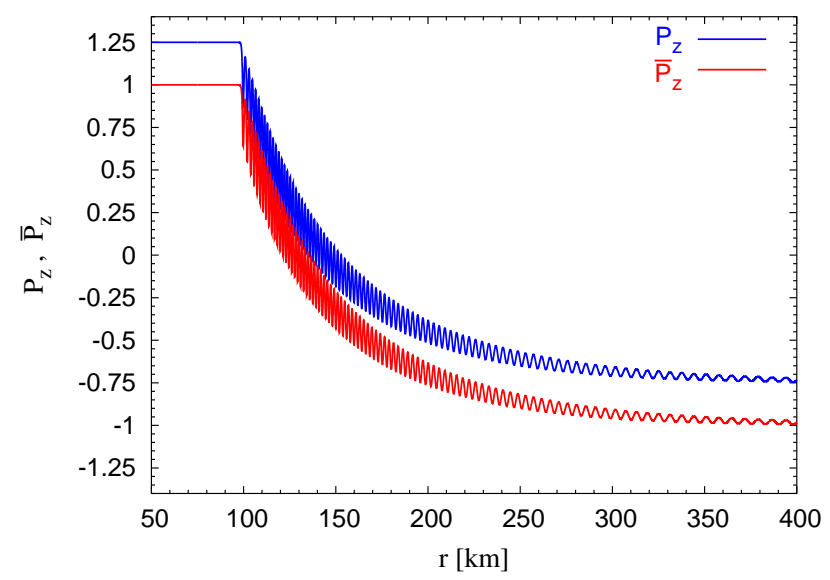

FIG. 1: Schematic evolution of the $z$-components of the total polarization vectors for neutrinos and antineutrinos in a SN caused by neutrino-neutrino interactions for the invertedhierarchy example described in the text. 
visible evolution takes place.

Flavor oscillations do not change those parts of the flavor fluxes that are already equal, only the transformation of the excess $\bar{\nu}_{e}$ flux over the $\bar{\nu}_{x}$ flux is observable, and likewise for neutrinos. The polarization vectors only represent this excess. Therefore, without loss of generality we may set $F_{\nu_{x}}=F_{\bar{\nu}_{x}}=0$ in our examples or equivalently, we may picture $F_{\nu_{e}}$ and $F_{\bar{\nu}_{e}}$ to represent $F_{\bar{\nu}_{e}}-F_{\bar{\nu}_{x}}$ and likewise for neutrinos. Our chosen parameters mean that at the neutrino sphere $(R=10 \mathrm{~km})$ the excess of the $\nu_{e}$ flux over the $\nu_{x}$ flux is $25 \%$ larger than the excess of the $\bar{\nu}_{e}$ flux over the $\bar{\nu}_{x}$ flux $(\epsilon=0.25) . \bar{P}_{z}=+1$ then represents a pure $\bar{\nu}_{e}$ excess flux, $\bar{P}_{z}=0$ represents equal excess fluxes of both flavors, and $\bar{P}_{z}=-1$ a pure $\bar{\nu}_{x}$ excess flux, and analogous for $\nu_{e}$ with $1 \rightarrow 1+\epsilon$.

The main features of Fig. 1 are nicely explained after recognizing that the equations of motion can be brought into a form where they are equivalent to a gyroscopic pendulum [7, 9]. The initial "plateau phase" corresponds to synchronized oscillations or, in the pendulum language, to a fast precession. We call the radius where this phase ends the synchronization radius $r_{\text {synch }}$. The decline with "wiggles" represents a nutation mode. The overall decline is caused by the dilution of the neutrino flux and their increasing collinearity with distance, corresponding to a decline of their effective interaction energy.

One salient feature of Fig. [1 is that the $\bar{\nu}_{e}$ flux completely converts to $\bar{\nu}_{x}$, whereas the $\nu_{e}$ flux converts to $\nu_{x}$ only to the extent allowed by the conservation of $P_{z}-\bar{P}_{z}=\epsilon$. This conservation is exact in the mass basis that approximately coincides with the interaction basis if the mixing angle is small. In other words, only $\nu_{e} \bar{\nu}_{e}$ pairs convert to $\nu_{x} \bar{\nu}_{x}$ pairs, whereas the unpaired $\nu_{e}$ excess remains in its original flavor [7].

The current-current nature of the weak interaction causes the interaction energy to depend on $(1-\cos \theta)$ for two trajectories with relative angle $\theta$. Therefore, neutrinos emitted in different directions from a SN core experience different refractive effects [5, 6]. As a result, one would expect that their flavor content evolves differently, leading to kinematical decoherence between different angular modes [2]. Two of us have recently shown that this multi-angle decoherence is indeed unavoidable in a "symmetric gas" of equal densities of neutrinos and antineutrinos [8]. Moreover, this effect is self-accelerating in that an infinitesimal anisotropy is enough to trigger an exponential run-away towards flavor equipartition, both for the normal and inverted hierarchies.

In the SN context, however, it has been numerically observed that the evolution is much more similar to the single-angle (or the isotropic) case [ $[5,6]$. The flux emitted by a $\mathrm{SN}$ is extremely anisotropic. If one assumes $\nu \bar{\nu}$ symmetry, flavor decoherence is swift and unavoidable. Therefore, the observed suppression of multi-angle decoherence must be related to the $\nu_{e} \bar{\nu}_{e}$ asymmetry that is generated by SN core deleptonization.

To illustrate this point we show in Fig. 2 a few examples along the lines of Fig. 1 but now for multi-angle emission from the neutrino sphere that is again taken at $10 \mathrm{~km}$. We consider different values of an asymmetry parameter that we define as

$$
\epsilon=\frac{F\left(\nu_{e}\right)-F\left(\nu_{x}\right)}{F\left(\bar{\nu}_{e}\right)-F\left(\bar{\nu}_{x}\right)}-1=\frac{F\left(\nu_{e}\right)-F\left(\bar{\nu}_{e}\right)}{F\left(\bar{\nu}_{e}\right)-F\left(\bar{\nu}_{x}\right)},
$$

where we have used $F\left(\nu_{x}\right)=F\left(\bar{\nu}_{x}\right)$. As mentioned earlier, we can assume $F\left(\nu_{x}\right)=F\left(\bar{\nu}_{x}\right)=0$ at the neutrino sphere without loss of generality. The left panels are for the normal hierarchy, the right panels for the inverted hierarchy.

$P_{z}(r)-\bar{P}_{z}(r)=\epsilon$ is constant so that it is sufficient to show $\bar{P}_{z}(r)$ alone. However, the length $\bar{P}=|\overline{\mathbf{P}}|$ is no longer preserved: Complete kinematical decoherence among the angular modes would cause $\bar{P}=0$. On the other hand, if $\bar{P}=1$ remains fixed, this signifies that all modes evolve coherently with each other. We use $\overline{\mathbf{P}}$ rather than $\mathbf{P}$ because the former measures what happens to the $\nu_{e} \bar{\nu}_{e}$ pairs, whereas the latter also includes the conserved $\nu_{e}$ excess.

In the top row we use $\epsilon=0$ (symmetric case). The flavor content decoheres quickly as expected. Both the length and the $z$-components of $\mathbf{P}$ and $\overline{\mathbf{P}}$ shrink to zero within about 20 meters of the nominal neutrino sphere.

On the other extreme, we show in the bottom row the same for $\epsilon=0.25$. In the normal hierarchy, nothing visible happens, in analogy to the single-angle case. In the inverted hierarchy, the transformation is similar, but not identical, to the single-angle case. The nutations wash out quickly. Shortly after exiting from the synchronization phase, the length $\bar{P}$ shrinks a bit, but stays almost constant thereafter. Clearly, some sort of multi-angle effect has happened as we will discuss further in Sec. III, but multi-angle decoherence has certainly not occurred.

In the two middle rows we show intermediate cases with $\epsilon=0.06$ and 0.12 , respectively. For the inverted hierarchy, these examples are qualitatively equivalent. The evolution is at first similar to the single-angle case and analogous to $\epsilon=0.25$. The nutations are washed out and the length $\bar{P}$ shrinks a little bit after the synchronization radius. At some larger radius, however, something new happens in that $\bar{P}$ suddenly shrinks significantly, although not to zero, and there is a distinct feature in the evolution of the $z$-component. Now we obtain partial decoherence. The final flavor content is very different from the single-angle case.

In the normal hierarchy, and for $\epsilon=0.06$, we obtain large decoherence that begins abruptly at some radius far beyond $r_{\text {synch }}$. For the larger asymmetry $\epsilon=0.12$, the length $\bar{P}$ also shrinks, but closely tracks $\bar{P}_{z}$. As we will see, this case is somewhat like Phase II of the invertedhierarchy case, i.e., a certain amount of shrinking of the length of $\bar{P}$ and thus a clear multi-angle effect, but no real decoherence.

Depending on the deleptonization flux, here represented by the asymmetry parameter $\epsilon$, the system behaves very differently. In particular, for the inverted hierarchy it is striking that there are either two or three 

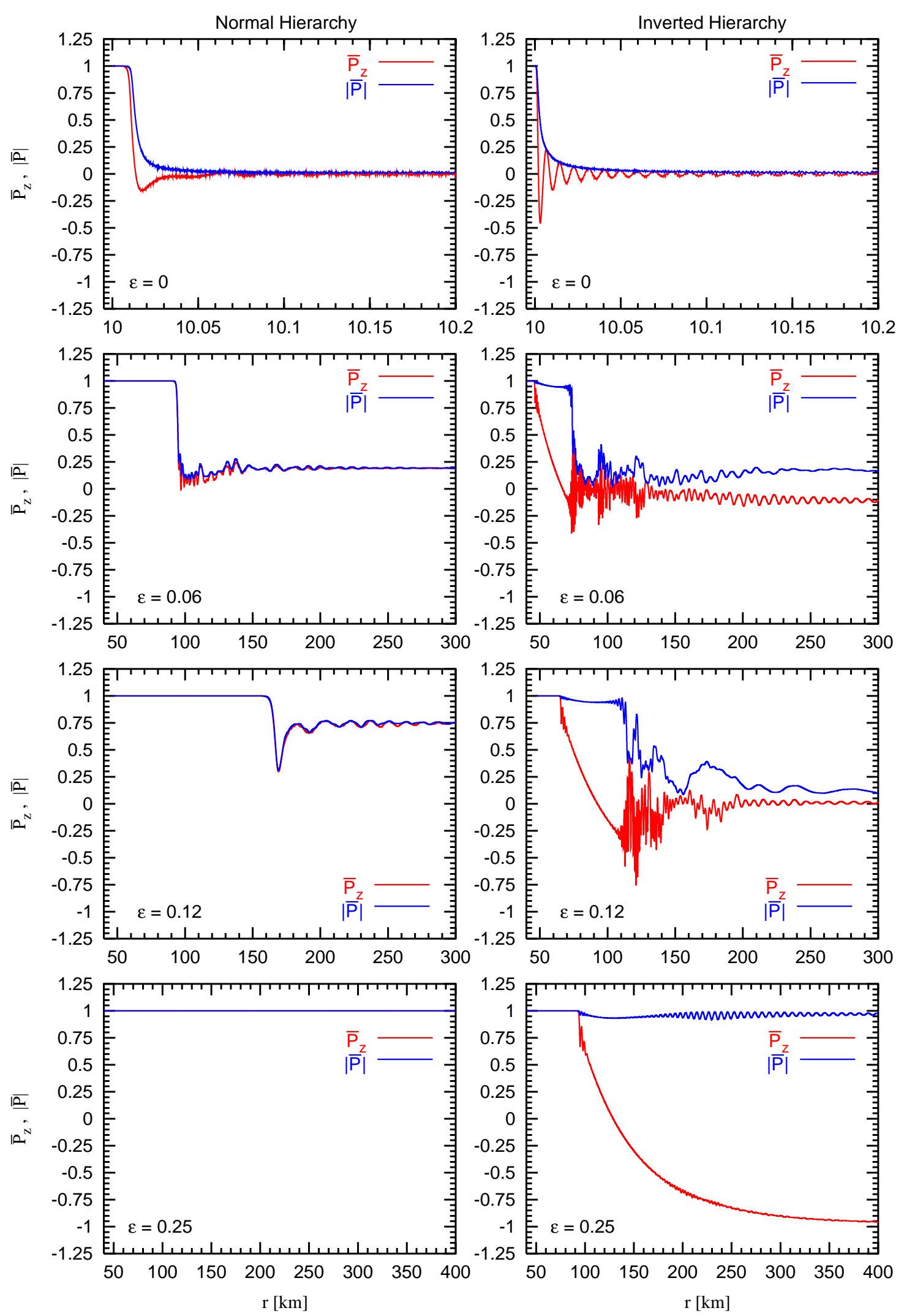

FIG. 2: Radial evolution of $\bar{P}_{z}$ in a schematic SN model as in Fig. 1 but now for multi-angle neutrino emission at the neutrino sphere $(R=10 \mathrm{~km})$. In addition we show the length $\bar{P}=|\overline{\mathbf{P}}|$ as a measure of kinematical coherence. Left: normal hierarchy. Right: inverted hierarchy. From top to bottom: $\epsilon=0,0.06,0.12$ and 0.25 , where $\epsilon$ is defined in Eq. (1). 
distinct phases. We always have the initial synchronized phase at large neutrino densities. Next, there is always the quasi single-angle pair-transformation phase at distances larger than $r_{\text {synch }}$. Just beyond this radius, the global polarization vectors quickly shrink by a small amount, but then stabilize immediately. Finally, if $\epsilon$ is below some critical value, there is a sharp transition to a third phase where the different angular modes decohere significantly, but not completely. The practical outcome for the flavor fluxes emerging from the dense-neutrino region is very different depending on $\epsilon$. The transition between these regimes is abrupt, a small change of $\epsilon$ is enough to cause one or the other form of behavior.

While these phenomena call for an analytic quantitative understanding, we are here less ambitious, but more practical. We study numerically for which range of parameters the different forms of behavior occur. Towards this goal we first set up, in Sec. II our conventions, the equations of motion for a spherically symmetric system, and establish the connection between the parameters of our schematic model with those of a realistic SN scenario. In Sec. III we describe in more detail what happens in the different phases of evolution diagnosed in Fig. 2] and identify useful measures of decoherence. In Sec. IV we investigate the role of our various model parameters in determining if the system kinematically decoheres. We discuss our findings and conclude in Sec. V. In Appendix A we derive the equations of motion adapted to spherical symmetry.

\section{SETUP OF THE PROBLEM}

\section{A. Equations of motion}

To study the flavor evolution of the neutrino flux emitted by a SN core we solve numerically the equations of motion for the flavor-dependent number fluxes, assuming spherical symmetry. We always work in a two-flavor scenario between $\nu_{e}$ and another flavor $\nu_{x}$, characterized by the atmospheric $\Delta m^{2}$ and by a vacuum mixing angle $\theta$ that is taken to represent the unknown 13-mixing angle.

Our fundamental quantities are the flux matrices in flavor space $\mathrm{J}_{r}$ that depend on the radial coordinate $r$ (Appendix A). The diagonal entries represent the total neutrino number fluxes through a sphere of radius $r$. In the absence of oscillations, $J_{r}$ would not depend on the radius at all. The flux matrices are represented by polarization vectors $\mathbf{P}_{r}$ in the usual way,

$$
\mathrm{J}_{r}=\frac{F\left(\nu_{e}\right)+F\left(\nu_{x}\right)}{2}+\frac{F\left(\bar{\nu}_{e}\right)-F\left(\bar{\nu}_{x}\right)}{2} \mathbf{P}_{r} \cdot \boldsymbol{\sigma}
$$

$$
\overline{\mathrm{J}}_{r}=\frac{F\left(\bar{\nu}_{e}\right)+F\left(\bar{\nu}_{x}\right)}{2}+\frac{F\left(\bar{\nu}_{e}\right)-F\left(\bar{\nu}_{x}\right)}{2} \overline{\mathbf{P}}_{r} \cdot \boldsymbol{\sigma},
$$

where $\boldsymbol{\sigma}$ is the vector of Pauli matrices. Antineutrino quantities are always denoted with an overbar. The number fluxes $F(\nu)$ are understood at the neutrino sphere. In both equations the term proportional to the polarization vector is normalized to the antineutrino flux. As a consequence, at the neutrino sphere we have the normalization

$$
P=|\mathbf{P}|=1+\epsilon \quad \text { and } \quad \bar{P}=|\overline{\mathbf{P}}|=1 .
$$

In this way, we treat the excess flux from deleptonization as an adjustable parameter without affecting the baseline flux of antineutrinos.

The diagonal part of the flux matrices is conserved and irrelevant for flavor oscillations. The polarization vector $\mathbf{P}_{r}$ only captures the difference between the flavor fluxes. For this reason we have defined the asymmetry $\epsilon$ in terms of the flux differences.

Multi-angle effects are at the focus of our study. We label different angular modes with

$$
u=\sin ^{2} \vartheta_{R}
$$

where $\vartheta_{R}$ is the zenith angle at the neutrino sphere $r=R$ of a given mode relative to the radial direction. The parameter $u$ is fixed for every trajectory whereas the physical zenith angle $\vartheta_{r}$ at distance $r$ varies. Therefore, using the local zenith angle to label the modes would complicate the equations.

We will consider two generic angular distributions for the modes. In the multi-angle case we assume that the neutrino radiation field is "half isotropic" directly above the neutrino sphere, i.e., all outward moving modes are equally occupied as expected for blackbody emission. This implies (Appendix A)

$$
\mathbf{P}_{u, r}=\mathrm{d} \mathbf{P}_{r} / \mathrm{d} u=\text { const. }
$$

at $r=R$ for $0 \leq u \leq 1$. Note that $u=0$ represents radial modes, $u=1$ tangential ones. The other generic distribution is the single-angle case where all neutrinos are taken to be launched at $45^{\circ}$ at the neutrino sphere so that $u=1 / 2$ for all neutrinos.

For a monochromatic energy distribution, the equations of motion in spherical symmetry are (Appendix A) 


$$
\begin{aligned}
& \partial_{r} \mathbf{P}_{u, r}=+\frac{\omega \mathbf{B} \times \mathbf{P}_{u, r}}{v_{u, r}}+\frac{\lambda_{r} \mathbf{L} \times \mathbf{P}_{u, r}}{v_{u, r}}+\mu \frac{R^{2}}{r^{2}}\left[\left(\int_{0}^{1} \mathrm{~d} u^{\prime} \frac{\mathbf{P}_{u^{\prime}, r}-\overline{\mathbf{P}}_{u^{\prime}, r}}{v_{u^{\prime}, r}}\right) \times\left(\frac{\mathbf{P}_{u, r}}{v_{u, r}}\right)-\left(\mathbf{P}_{r}-\overline{\mathbf{P}}_{r}\right) \times \mathbf{P}_{u, r}\right], \\
& \partial_{r} \overline{\mathbf{P}}_{u, r}=-\frac{\omega \mathbf{B} \times \overline{\mathbf{P}}_{u, r}}{v_{u, r}}+\frac{\lambda_{r} \mathbf{L} \times \overline{\mathbf{P}}_{u, r}}{v_{u, r}}+\mu \frac{R^{2}}{r^{2}}\left[\left(\int_{0}^{1} \mathrm{~d} u^{\prime} \frac{\mathbf{P}_{u^{\prime}, r}-\overline{\mathbf{P}}_{u^{\prime}, r}}{v_{u^{\prime}, r}}\right) \times\left(\frac{\overline{\mathbf{P}}_{u, r}}{v_{u, r}}\right)-\left(\mathbf{P}_{r}-\overline{\mathbf{P}}_{r}\right) \times \overline{\mathbf{P}}_{u, r}\right],
\end{aligned}
$$

where the radial velocity of mode $u$ at radius $r$ is

$$
v_{u, r}=\sqrt{1-u R^{2} / r^{2}} .
$$

Further, $\omega=\left|\Delta m^{2} / 2 E\right|$ is the vacuum oscillation frequency, taken to be positive. $\mathbf{B}=(\sin 2 \theta, 0, \pm \cos 2 \theta)$ where the mixing angle $\theta$ is usually taken to be small. $B_{z}<0$ corresponds to the normal hierarchy, $B_{z}>0$ to the inverted hierarchy. $\mathbf{L}$ is a unit vector in the $z$ direction because we work in the interaction basis. The matter density is represented by

$$
\lambda_{r}=\sqrt{2} G_{\mathrm{F}}\left[n_{e^{-}}(r)-n_{e^{+}}(r)\right] .
$$

The strength of the neutrino-neutrino interaction is parameterized by

$$
\mu=\sqrt{2} G_{\mathrm{F}}\left(F_{\bar{\nu}_{e}}^{R}-F_{\bar{\nu}_{x}}^{R}\right),
$$

where the fluxes are taken at the neutrino sphere with radius $R$.

The somewhat complicated structure of the equations arises from projecting the evolution of each mode on the radial direction. This is still very much simpler than following the evolution on every trajectory as a function of distance (or time) on that trajectory. We have here a closed set of differential equations that is not hard to solve numerically.

We show in Appendix $\mathrm{A}$ that for $r \gg R$, the vacuum and matter oscillation terms take on the familiar planewave form because at large distances all neutrinos essentially move on radial trajectories. The neutrino-neutrino term falls off as $r^{-4}$, in agreement with the previous literature.

\section{B. Schematic supernova model}

We always consider a two-flavor oscillation scenario driven by the atmospheric $\Delta m^{2}=1.9-3.0 \times 10^{-3} \mathrm{eV}^{2}$. Assuming $\left\langle E_{\nu}\right\rangle=15 \mathrm{MeV}$, the oscillation frequency is $\omega=0.3-0.5 \mathrm{~km}^{-1}$. To be specific, we use

$$
\omega=\left\langle\frac{\Delta m^{2}}{2 E}\right\rangle=0.3 \mathrm{~km}^{-1}
$$

as a benchmark value in the monochromatic model.

The total energy output of a SN is around $3 \times 10^{53} \mathrm{erg}$, corresponding to $0.5 \times 10^{53} \mathrm{erg}$ in each of the six neutrino species if we assume approximate equipartition of the
TABLE I: Default values for our model parameters.

\begin{tabular}{lll}
\hline \hline Parameter & Standard value & Definition \\
\hline$\epsilon$ & 0.25 & Eq. (1) \\
$\mu$ & $7 \times 10^{5} \mathrm{~km}^{-1}$ & Eq. (9) \\
$\omega$ & $0.3 \mathrm{~km}^{-1}$ & Eq. (10) \\
$\sin 2 \theta$ & $10^{-3}$ & - \\
\hline \hline
\end{tabular}

emitted energy. If this energy is emitted over $10 \mathrm{~s}$, the average luminosity per flavor would be $0.5 \times 10^{52} \mathrm{erg} / \mathrm{s}$. However, at early times during the accretion phase, the luminosity in the $\bar{\nu}_{e}$ flavor can exceed $3 \times 10^{53} \mathrm{erg} / \mathrm{s}[13]$. As our baseline estimate we use

$$
\begin{aligned}
\mu & =7 \times 10^{5} \mathrm{~km}^{-1} \\
& \times\left(\frac{L_{\bar{\nu}_{e}}}{\left\langle E_{\bar{\nu}_{e}}\right\rangle}-\frac{L_{\bar{\nu}_{x}}}{\left\langle E_{\bar{\nu}_{x}}\right\rangle}\right) \frac{15 \mathrm{MeV}}{10^{52} \mathrm{erg} / \mathrm{s}}\left(\frac{10 \mathrm{~km}}{R}\right)^{2} .
\end{aligned}
$$

This is significantly larger than the assumptions of previous studies [5, 7]. Unless otherwise stated, we always use the benchmark values for the different parameters summarized in Table प.

In our calculations we always take the neutrino sphere at the radius $R=10 \mathrm{~km}$. Of course, the physical neutrino sphere is not a well-defined concept. Therefore, the radius $R$ simply represents the location where we fix the inner boundary condition. However, essentially nothing happens until the synchronization radius $r_{\text {synch }} \gg R$ because the in-medium mixing angle is extremely small and both neutrinos and antineutrinos simply precess around $\mathbf{B}$. Therefore, as far as the vacuum and matter oscillation terms are concerned, it is almost irrelevant where we fix the inner boundary condition.

Not so for the neutrino-neutrino term because we also fix the angular distribution at $r=R$. While the $r^{-2}$ scaling from flux dilution is unaffected by the radius for the inner boundary condition, the "collinearity suppression" also scales as $(R / r)^{2}$ for $r \gg R$. If we fix a half-isotropic distribution or a single angle of $45^{\circ}$ at a larger radius $R^{\prime}$, the new inner boundary condition essentially amounts to $\mu \rightarrow \mu^{\prime}=\mu\left(R^{\prime} / R\right)^{2}$. In the early phase after bounce $R^{\prime}=30 \mathrm{~km}$ could be more realistic, leading to a $\mu$ value almost an order of magnitude larger. Evidently, $\mu$ is a rather uncertain model parameter that can differ by orders of magnitude from our benchmark value.

However, collective pair conversions only begin at $r_{\text {synch }}$ where $\mu$ is so small that synchronization ends. 
Therefore, the main impact of a modified $\mu$ is to change $r_{\text {synch }}$ and thus to push the collective pair conversions to larger radii. The oscillations are synchronized if [7]

$$
\frac{\mu}{\omega}>\frac{2}{(1-\sqrt{1+\epsilon})^{2}} .
$$

In our single-angle case we find from Eq. A20) that the effective neutrino-neutrino interaction strength varies at large distances as

$$
\mu_{\mathrm{eff}}(r)=\mu \frac{R^{4}}{2 r^{4}} .
$$

Therefore, the synchronization radius is

$$
\begin{aligned}
\frac{r_{\mathrm{synch}}}{R} & =\left(\frac{\sqrt{1+\epsilon}-1}{2}\right)^{1 / 2}\left(\frac{\mu}{\omega}\right)^{1 / 4} \\
& \approx \frac{\sqrt{\epsilon}}{2}\left(\frac{\mu}{\omega}\right)^{1 / 4} .
\end{aligned}
$$

The second line assumes $\epsilon \ll 1$. If we use our benchmark values $\omega=0.3 \mathrm{~km}^{-1}, \mu=7 \times 10^{5} \mathrm{~km}^{-1}, R=10 \mathrm{~km}$ and $\epsilon=0.25$, we find $r_{\text {synch }}=95 \mathrm{~km}$, corresponding well, for example, to Fig. 1. In any event, if $\mu$ is taken to be uncertain by two orders of magnitude, $r_{\text {synch }}$ only changes by a factor of 3 .

The total electron lepton number emitted from a collapsed SN core is about $3 \times 10^{56}$. On the other hand, assuming that each neutrino species carries away $0.5 \times 10^{53} \mathrm{erg}$ with an average energy of $15 \mathrm{MeV}$, the SN core emits about $2 \times 10^{57}$ neutrinos in each of the six species. In this simplified picture, the SN emits on average about $15 \%$ more $\nu_{e}$ than $\bar{\nu}_{e}$. However, in the oscillation context we need the excess of $F_{\nu_{e}}-F_{\nu_{x}}$ relative to the same quantity for antineutrinos as defined in Eq. (11). The true value of $\epsilon$ thus depends sensitively on the detailed fluxes and spectra of the emitted neutrinos. The asymmetry parameter is large when the first hierarchy in $F_{\nu_{e}}>F_{\bar{\nu}_{e}}>F_{\bar{\nu}_{x}}=F_{\nu_{x}}$ is large and/or the second hierarchy is small. Even if $F_{\bar{\nu}_{x}}$ is as small as half of $F_{\bar{\nu}_{e}}$, the asymmetry $\epsilon$ would be as large as $30 \%$, even when $F_{\nu_{e}}$ exceeds $F_{\bar{\nu}_{e}}$ by only $15 \%$.

\section{Numerical multi-angle decoherence and the inner boundary condition}

One important and somewhat confusing complication of numerically solving the equations of motion is the phenomenon of numerical multi-angle decoherence. To integrate Eq. (6) one needs to work with a finite number of angular modes, equivalent to coarse-graining the phase space of the system. If the number of angular bins is chosen smaller than some critical number $N_{\min }$, multiangle decoherence occurs for $r<r_{\text {synch }}$, where physically it is not possible and does not occur for a fine-grained calculation. This phenomenon is shown, for example, in Fig. 3 of Ref. [5]. It is not caused by a lack of numerical precision, but a result of the coarse-graining of phase space. A related phenomenon is recurrence as discussed in the context of multi-angle decoherence in Ref. [8].

In other words, a coarsely grained multi-angle system behaves differently than a finely grained one. A smaller mixing angle reduces $N_{\text {min }}$, a larger neutrino-neutrino interaction strength increases it. It should be possible to estimate $N_{\text {min }}$ from first principles, but for the moment we need to rely on trial and error.

Starting the integration at $r=R$ is doubly punishing because the fast oscillations of individual modes caused by a large $\mu$ requires many radial steps for the numerical integration and avoiding numerical decoherence requires a large number of angular modes. On the other hand, in this region nothing but fast synchronized oscillations take place that have no physical effect if the mixing angle is small. Using a larger radius as a starting point for the integration avoids both problems and does not modify the overall flavor evolution at larger distances.

From the physical perspective, the "neutrino sphere" is not a well-defined concept because different energy modes and different species decouple at different radii, and in any case, each individual neutrino scatters last at a different radius. If the exact inner boundary condition would matter, we would need to solve the full kinetic equations, including neutral-current and charged-current collisions. It is the beauty of the neutrino-neutrino flavor transformation problem that the real action begins at $r_{\text {synch }}$, significantly outside the neutrino sphere. Our approach of reducing the equations of motion to the refractive terms is only self consistent because the exact location of the inner boundary condition is irrelevant.

In summary, the nominal neutrino sphere at $R=$ $10 \mathrm{~km}$ is nothing but a point of reference where we normalize the fluxes and fix the angular distribution. As a starting point for integration we typically use $r_{0}=$ $0.75 r_{\text {synch. }}$. A few hundred angular modes are then usually enough to avoid numerical decoherence.

We note, however, that the normal-hierarchy cases are more sensitive to both the number of angular bins and the starting radius for the integration. It can happen that a case that looks like the $\epsilon=0.12$ example in Fig. 2, which shows a mild shrinking of the polarization vector, can become "more coherent" by choosing a smaller starting radius which then may also require a larger number of modes. For the normal hierarchy, the different multiangle cases are less cleanly separated from each other than in the inverted hierarchy in that the transition is less abrupt as a function of $\epsilon$.

When physical multi-angle decoherence occurs (e.g. the middle rows of Fig. 22), a much larger number of modes is needed to provide reproducible results. However, we are here not interested in the exact final outcome, we are mostly interested in the range of parameters that lead to decoherence. Therefore, massive computation power is not needed for our study.

For those cases where we include a non-trivial spectrum of energies we also need energy bins. A distribution of energies does not lead to kinematical decoherence in 
the context of collective neutrino oscillations [7] so that the number of energy bins is not a crucial parameter. Of course, to resolve the energy-dependent behavior and especially the spectral splits [5, 9, 10, 11], a sufficiently fine-grained binning is required. It provides better resolution, but not a qualitatively different form of behavior.

\section{COHERENT EVOLUTION VS. DECOHERENCE}

\section{A. Different forms of evolution}

Before investigating the conditions for decoherence among angular neutrino modes we first take a closer look at what happens in the different cases shown in Fig. 2. Considering first the quasi single-angle case with the asymmetry $\epsilon=0.25$, some insight is gained by looking at the final state of the evolution at some large radius where the neutrino-neutrino effects have completely died out and all modes simply perform vacuum oscillations. In the left-hand panels of Fig. 3] we show the end state of 500 polarization vectors, representing modes uniformly spaced in the angular coordinate $u$. In the upper panel we show the final state in the $x$ - $z$-plane ("side view"), in the lower panel in the $x$ - $y$-plane ("top view").

Initially, all polarization vectors are aligned in the flavor direction. At the beginning of the pair transformation phase at $r_{\text {synch }}$, some are peeled off, forming a spiral structure that is easily gleaned from the left panels of Fig. 3. This structure continues to evolve almost as in the single-angle case, i.e., once established it moves almost like a rigid body and eventually orients itself in the negative $\mathbf{B}$-direction. Of course, it continues to rotate around the $\mathbf{B}$ direction even at large radii because of vacuum oscillations.

The spiral structure is different depending on the mixing angle. We illustrate this in Fig. 4 where we show the top view in analogy to the lower-left panel of Fig. 3 for different choices of mixing angle. For a large $\sin 2 \theta$, the polarization vectors stay close to each other. For a smaller $\sin 2 \theta$, the spiral spreads over a larger solid angle and has more windings. We recall that a smaller $\sin 2 \theta$ also has the effect of causing a larger nutation depth of the flavor pendulum [8].

Now turn to the quasi decoherent case with $\epsilon=0.12$. Initially the same happens, but at the "decoherence radius" the spiral structure dissolves almost instantaneously. The polarization vectors enter a complicated structure as illustrated by the end state (central panels of Fig. 3). Moreover, they are spread out all over the unit sphere, having both positive and negative $z$-components. This structure looks different for different choices of $\sin 2 \theta$ and $\epsilon$. However, once a sufficient number of polarization vectors is used, it is reproducible. For $\epsilon=0.06$ the picture would be qualitatively similar.

Finally we show the fully symmetric case $(\epsilon=0)$ in the right-hand panels. Here decoherence is fast and com- plete. For a small mixing angle, all polarization vectors are confined to the $x$-z-plane. They distribute themselves on a circle in that plane.

For the normal hierarchy, we show in Fig. 5 as an explicit example the $\epsilon=0.12$ case of Fig. 2 that showed a clear multi-angle effect without strong decoherence. Once more we find a spiral structure. Most polarization vectors remain oriented roughly in their original direction, but in this case also with a tail of a few polarization vectors reversed. The quasi decoherent case $(\epsilon=0.06)$ and the symmetric system produce similar final pictures as the corresponding cases of the inverted hierarchy.

\section{B. Measures of decoherence}

Even in the quasi-decoherent cases the unit sphere is not uniformly filled with polarization vectors. Rather, in the mono-energetic case considered here, the occupied phase space is a one-dimensional subspace of the unit sphere. It is parameterized by the angular variable $u$ and shows a clear line-like structure. This picture suggests to use the length of this line on the unit sphere as another global measure besides the length $\bar{P}$ to discriminate between different modes of evolution [8]. In a numerical run with discrete angular bins, this quantity is simply the sum of the angles between neighboring polarization vectors. In Fig. 6] we show this quantity for the indicated values of $\epsilon$ as a function of radius for our inverted-hierarchy examples.

At the radius $r_{\text {synch }}$ where the spiral forms, the length on the unit sphere quickly increases from 0 to a value that is almost independent of $\epsilon$, but depends on the mixing angle. For smaller $\sin 2 \theta$ it is larger, corresponding to the spiral having more windings as indicated earlier. Later, this length stays practically constant, reflecting that the spiral structure, once established, does not change much except tilting toward the negative $\mathbf{B}$-direction and precessing around it.

When $\epsilon$ is smaller than a critical value, at the "decoherence radius" a sudden second growth phase shoots up from the plateau of these curves. For smaller $\epsilon$, the final length is longer, representing a more "phase-space filling" line on the unit sphere.

Note, however, that for $\epsilon$ close to zero, the line does not fill the unit sphere, but essentially stays in a narrow band. In the perfectly symmetric case, the motion of all polarization vectors is essentially confined to the $x-z$ plane, i.e., the polarization vectors distribute themselves over a great circle on the sphere as shown in the right panels of Fig. 3 . 

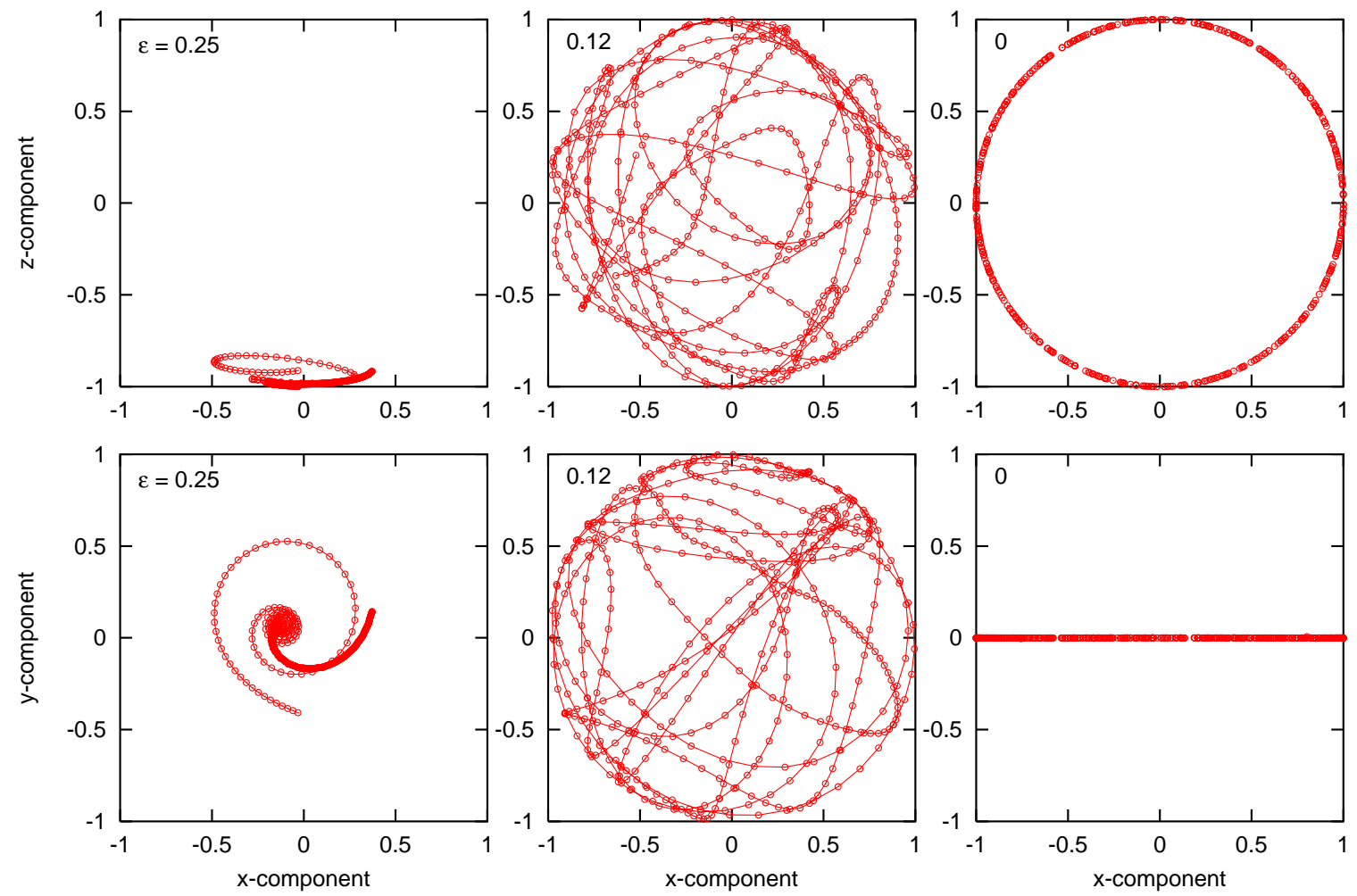

FIG. 3: Final location on the unit sphere of 500 antineutrino polarization vectors for our standard parameters and the inverted hierarchy. The top row is the "side view" ( $x$ - $z$-components), the bottom row the "top view" ( $x$ - $y$-components). Left: quasi single-angle case $(\epsilon=0.25)$. Middle: decoherent case $(\epsilon=0.12)$. Right: symmetric system $(\epsilon=0)$.
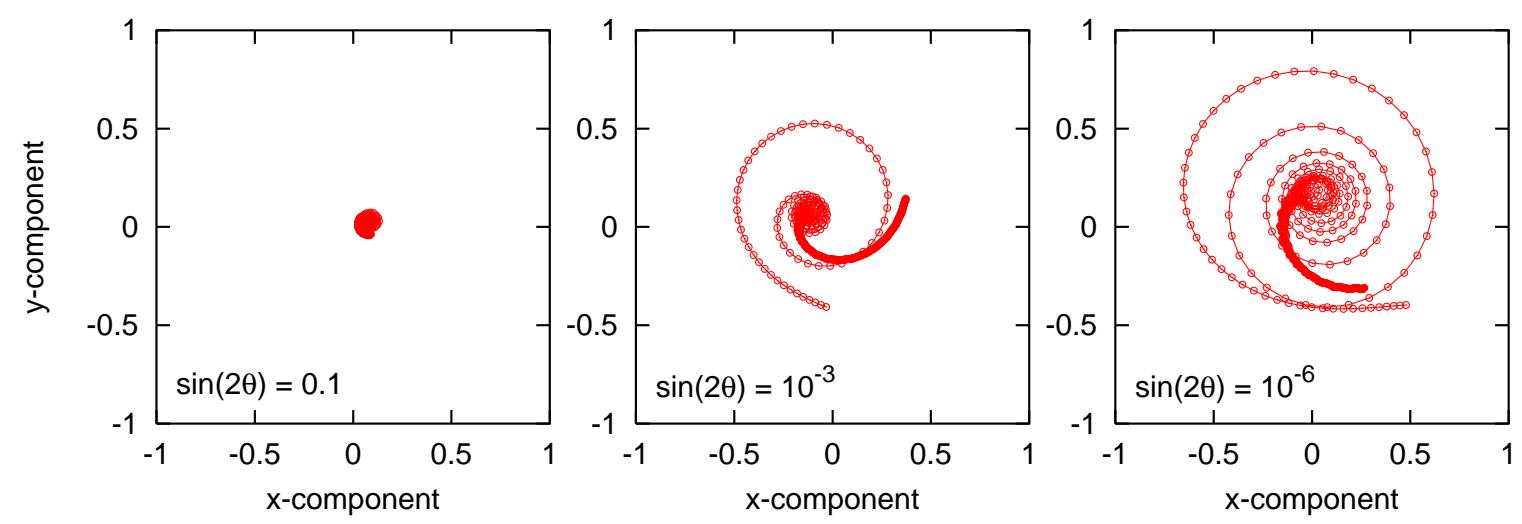

FIG. 4: Same as Fig. [3 now only top views for quasi single-angle cases with the mixing angles $\sin 2 \theta=0.1,10^{-3}$ and $10^{-6}$ from left to right. The middle panel is identical with the bottom left panel of Fig. 3 .

\section{ROLE OF MODEL PARAMETERS}

\section{A. Ordinary matter}

We now explore how various model parameters influence the behavior of the system. In the examples so far we have ignored matter because its effect is mainly to suppress the vacuum mixing angle. We here make this argument more precise. In Fig. 17 we show typical matter density profiles, expressed in terms of the matter oscil- lation frequency $\lambda(r)$, from numerical simulations of the Garching group for different times after collapse [14]. For comparison we also show $\mu(r)$ with $\mu(R)=7 \times 10^{5} \mathrm{~km}^{-1}$ and a radial variation in analogy to Eq. (A20).

We observe that for the shown density profiles, the line $\omega$ intersects $\lambda(r)$ at a radius far exceeding the denseneutrino region that lies within the radius where the $\mu(r)$ profile intersects $\omega$. In other words, the $\mathrm{H}$-resonance is far outside the region of interest except perhaps for very late times. Then, of course, the neutrino luminosity will be much smaller, i.e., the $\mu(r)$ curve would also shift down- 

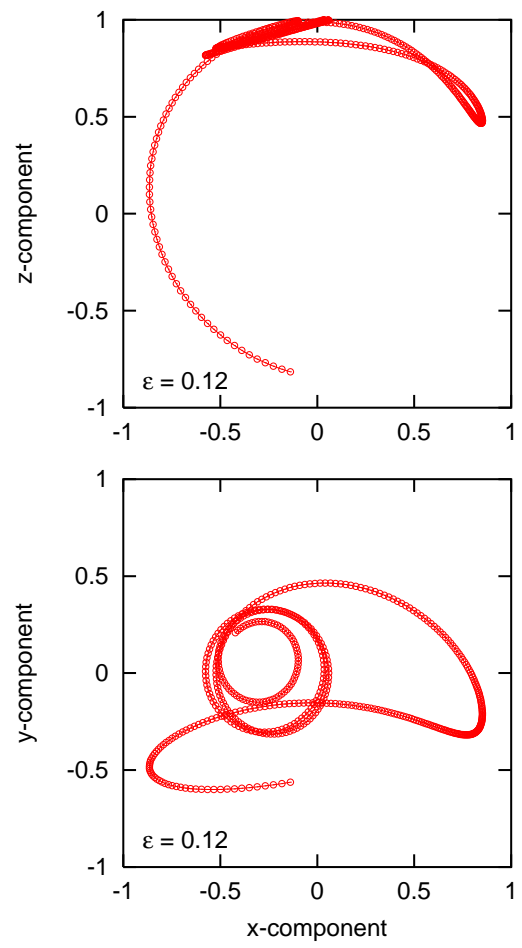

FIG. 5: Same as Fig. 3, here for the normal hierarchy and $\epsilon=0.12$.

ward and the dense-neutrino region would be limited to smaller radii.

The true density profiles may be much lower, especially at late times. This is even required for successful r-process nucleosynthesis. In this scenario an MSW resonance may take place within the dense-neutrino region, a case that was the focus of previous numerical studies [5, [6]. However, we will always assume that the

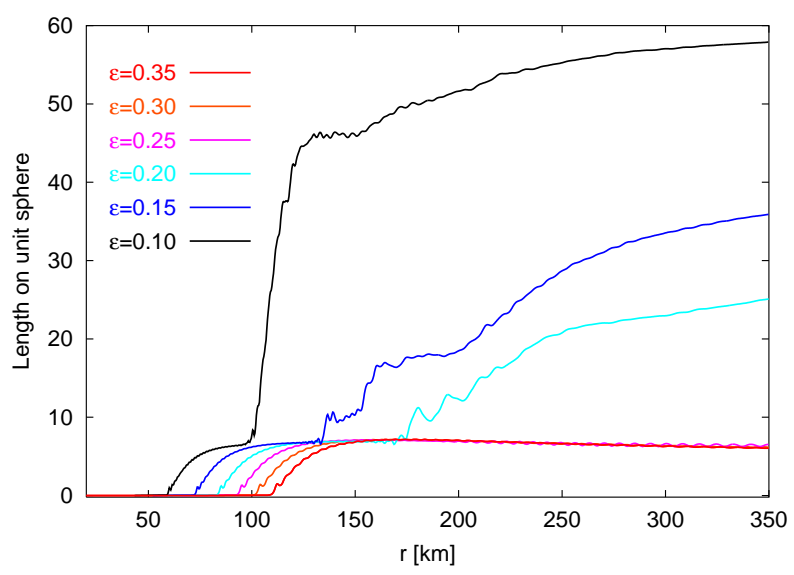

FIG. 6: Evolution of the length of the one-dimensional subspace occupied by the polarization vectors for our standard inverted hierarchy case, taking a series of different asymmetries $\epsilon$. The length grows to larger values for smaller asymmetries.
$\mathrm{H}$-resonance is at larger radii and that neutrino-neutrino refraction and ordinary matter effects do not interfere.

What is the impact of a large matter density in the region where neutrino-neutrino effects are important? In the previous literature it was recognized that a constant matter profile essentially reduces the effective mixing angle so that matter should have the same influence as a small vacuum mixing angle [4, 7]. We illustrate this point in Fig. 8 with the evolution of $\bar{P}_{z}$ for our usual case, but assuming now a large vacuum mixing angle $\sin 2 \theta=0.1$. In the synchronization region one can now see oscillations. We overlay this curve with $\bar{P}_{z}$, using the matter profile of Fig. 7 at $t=2 \mathrm{~s}$. As expected, matter has the effect of slightly delaying the onset of pair transformations and of increasing the depth of the nutation amplitude.

Actually, in the inverted hierarchy, the value of $\sin 2 \theta$ is only crucial at the onset of the bipolar oscillations. Once the overall polarization vector is tilted away from B, the initial "misalignment" with $\mathbf{B}$ no longer matters. Therefore, what is crucial for the role of matter is only its density around the region where synchronization ends. The in-medium mixing angle at $r_{\text {synch }}$ for the case shown in the top panel of Fig. 8 is $\sin 2 \theta_{\text {matter }}=3.35 \times 10^{-4}$, assuming $\sin 2 \theta_{\mathrm{vac}}=0.1$. Using this value of $\theta_{\text {matter }}$ as a vacuum mixing angle instead of matter yields the result shown in the bottom panel, again overlaid with the original vacuum case of $\sin 2 \theta=0.1$.

We conclude that indeed we can ignore matter entirely if we account for it schematically by a small vacuum mixing angle, at least in the inverted hierarchy. Moreover, the onset of collective pair transformations is only mildly changed by the choice of mixing angle. Its main impact is that it controls the depth of the nutation pattern. The exact matter profile is only important if it is so shallow that it causes an MSW resonance in the dense-neutrino region, a case that we do not investigate.

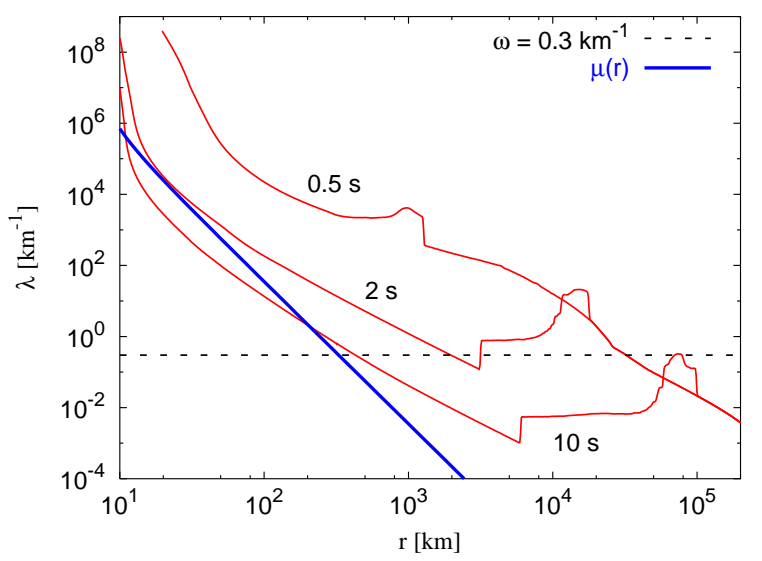

FIG. 7: Typical matter density profiles from numerical simulations of the Garching group at the indicated times after core bounce [14]. For comparison we also show our benchmark value $\omega=0.3 \mathrm{~km}^{-1}$ and $\mu(r)$ for a typical case. 


\section{B. Mixing angle}

This discussion suggests that, at least for the inverted hierarchy, the actual vacuum mixing angle does not strongly influence the issue of multi-angle decoherence because this effect happens when the global polarization vector is tilted far away from the $\mathbf{B}$ direction. On the other hand, we have already noted that the quasicoherent spiral structure that forms just beyond the synchronization radius has more windings for a smaller mixing angle so that the system is not identical.

To clarify the role of the mixing angle we have used our standard inverted-hierarchy case and have calculated the limiting asymmetry $\epsilon$ for decoherence for a broad range of mixing angles. We show the limiting contours in the plane of $\epsilon$ and $\sin 2 \theta$ in Fig. 9 for both hierarchies, above which multiangle decoherence does not appear.

We emphasize that the limiting $\epsilon$ shown in Fig. 9 has a different meaning for the two hierarchies. As discussed earlier, in the inverted hierarchy, $\bar{P}$ shortens somewhat even in the quasi single-angle regime. Therefore, as a for-
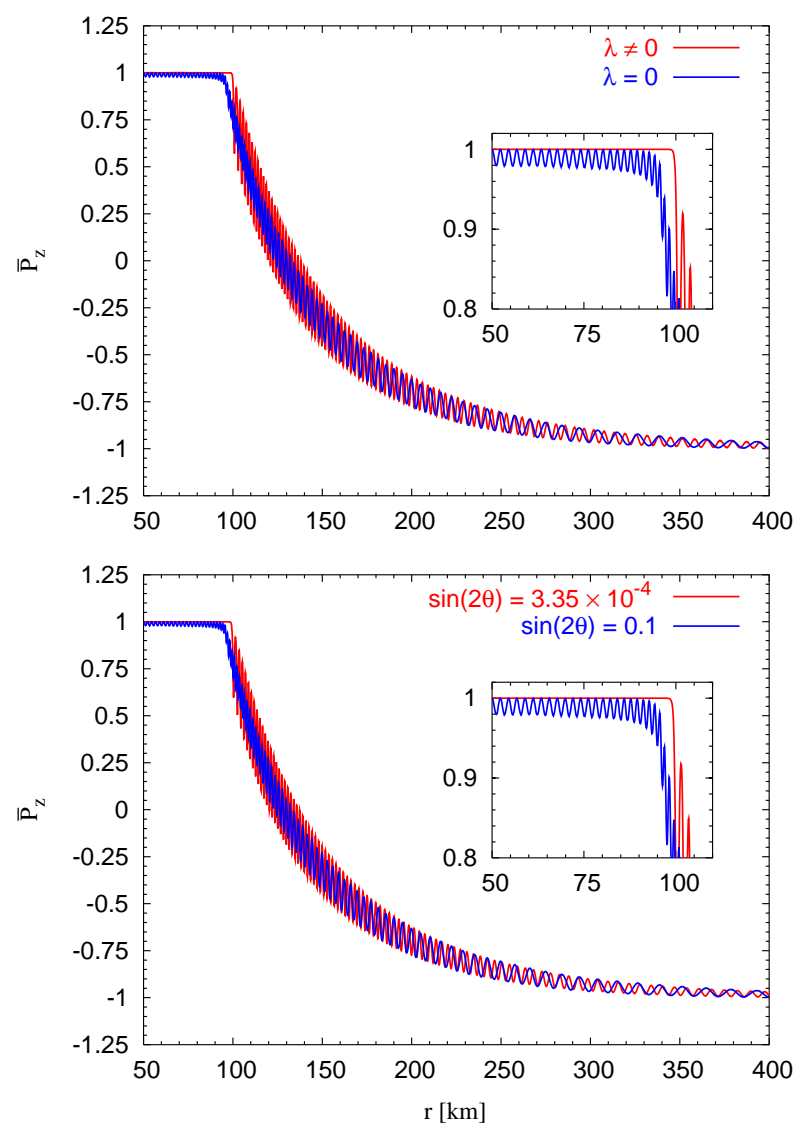

FIG. 8: Evolution for our standard inverted-hierarchy case for a large vacuum mixing angle of $\sin 2 \theta=0.1$ (blue/dotted lines in both panels), compared with different ways of suppressing the mixing angle (red/solid). Top: ordinary matter effect according to the profile at $t=2 \mathrm{~s}$ in Fig. 7 Bottom: small vacuum mixing angle of $\sin 2 \theta=3.35 \times 10^{-4}$.

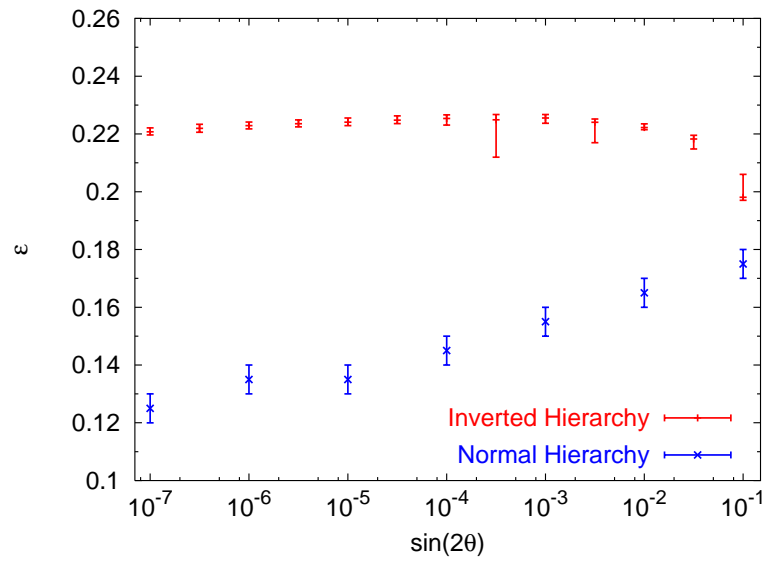

FIG. 9: Limiting $\epsilon$ for decoherence as a function of mixing angle for our standard example and both hierarchies.

mal criterion for distinguishing the regions of coherence and decoherence we use that the final $\bar{P}$ has shortened to less than 0.85 . The exact choice is irrelevant because the transition between the quasi-coherent and decoherent regimes is steep as a function of $\epsilon$.

Conversely, in the normal hierarchy, $\bar{P}$ need not visibly shorten at all as illustrated by the example in the lower left panel of Fig. 2. Therefore, we here demand that $\bar{P}$ does not visibly shorten in such a picture. We construct the demarcation line by decreasing $\epsilon$ in steps of 0.01 until the polarization vector for the first time shortens visibly. Finding this point requires a significant amount of manual iterations with a modified inner radius and number of angular bins to make sure the result does not depend on these numerical parameters. The error bars represent our confidence range for the true critical value.

We conclude that for the inverted hierarchy, multiangle decoherence is virtually independent from the value of $\sin 2 \theta$, except that for very large $\theta$ a slightly smaller asymmetry is enough to suppress decoherence. Assuming the presence of ordinary matter, such large mixing angles seem irrelevant, except perhaps at late times. Either way, it is conservative to assume a small mixing angle and we will use $\sin 2 \theta=10^{-3}$ as a default value.

For the normal hierarchy we find a strong dependence of the critical $\epsilon$ on $\log _{10}(\sin 2 \theta)$. For a smaller mixing angle it is easier to suppress decoherence. The normal hierarchy is very different from the inverted one in that for a small mixing angle, all polarization vectors stay closely aligned with the $z$-direction unless multi-angle decoherence takes place. Therefore, it is plausible that for a smaller mixing angle, decoherence effects are delayed.

\section{Energy distribution}

The neutrinos emitted from a SN core naturally have a broad energy distribution. In Ref. 8] it was noted that the energy distribution of neutrinos and antineutrinos is 

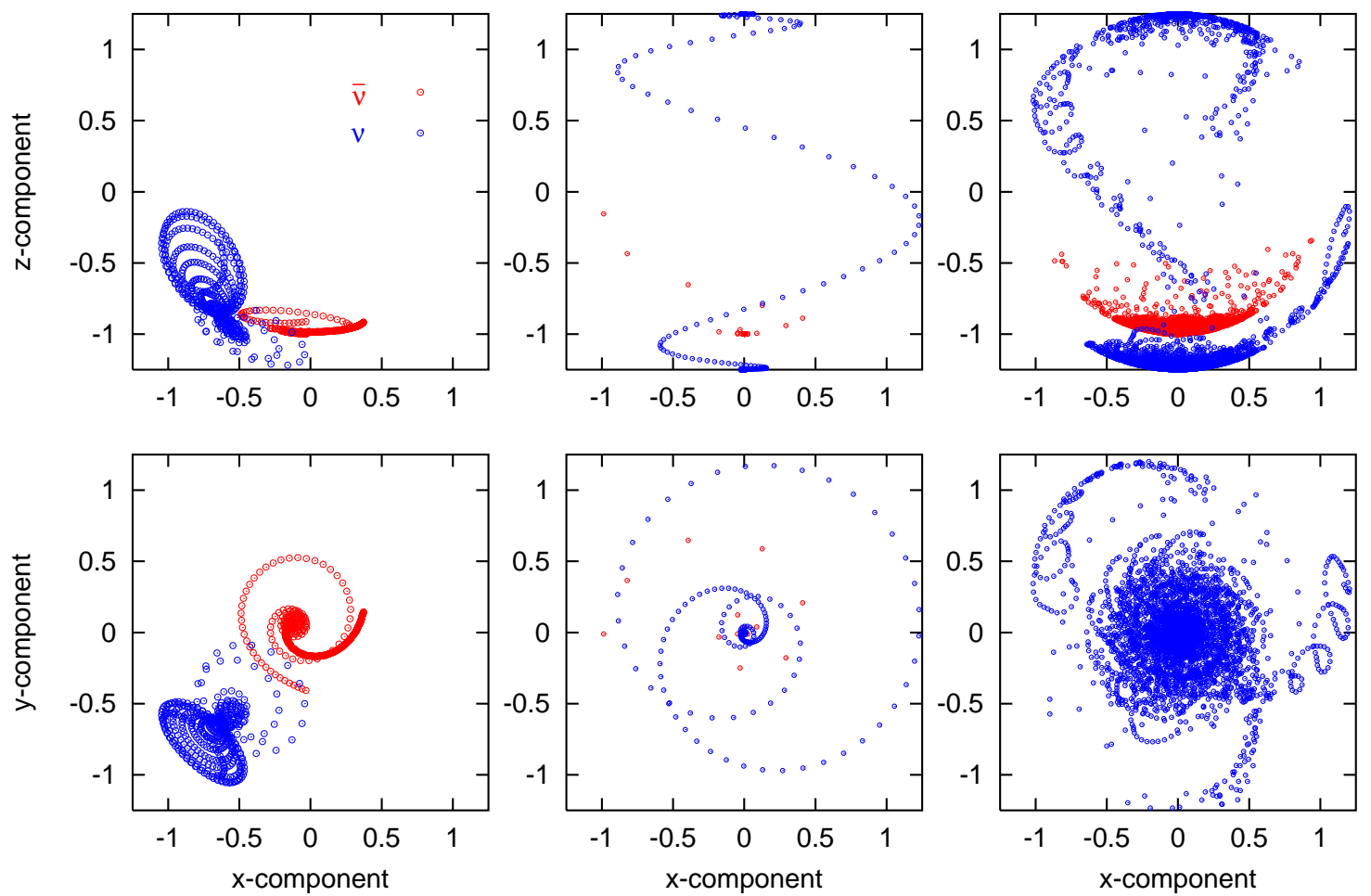

FIG. 10: Final state at a large radius of the polarization vectors for our standard parameters in analogy to Fig. 3 The antineutrinos (red/light gray) are on the unit sphere, whereas the neutrinos (blue/dark gray) live on a sphere of radius $1+\epsilon=1.25$. Left: monochromatic multi-angle, the antineutrinos being identical with the left column of Fig. 3. Middle: Box-like energy spectrum and single angle. Right: Box-like energy spectrum and multi angle. In the lower right panel we do not show the antineutrinos.

largely irrelevant for the question of decoherence as long as the oscillations exhibit self-maintained coherence [15]. The multi-angle transition to decoherence typically occurs within the dense-neutrino region where the synchronization of energy modes remains strong. Therefore, we expect that multi-angle decoherence is not significantly affected by the neutrino spectrum.

In order to compare a monochromatic system with one that has a broad energy distribution, the crucial quantity to keep fixed is not the average energy, but the average oscillation frequency $\langle\omega\rangle=\left\langle\Delta m^{2} / 2 E\right\rangle$. If we assume that neutrinos and antineutrinos have equal distributions, it is straightforward to adjust, for example, the temperature of a thermal distribution such that $\langle\omega\rangle$ is identical to our monochromatic standard case $\omega_{0}=0.3 \mathrm{~km}^{-1}$.

If we assume different distributions for neutrinos and antineutrinos, the equivalent $\omega_{0}$ is somewhat more subtle. Consider first two different monochromatic spectra for neutrinos with a fixed frequency $\omega_{1}$, and one for antineutrinos with a different frequency $\omega_{2}$ ("bichromatic system"). Following Ref. [4] one can return to a monochromatic situation by going into a reference frame that rotates around $\mathbf{B}$ with such a frequency that in vacuum $\mathbf{P}$ and $\overline{\mathbf{P}}$ precess around $\mathbf{B}$ with equal frequencies $\omega_{0}$, but in opposite directions. The rotation frequency for the corotating frame is $\omega_{\mathrm{c}}=\left(\omega_{1}-\omega_{2}\right) / 2$. Therefore, our bichromatic system behaves equivalently to a monochromatic one with $\omega_{0}=\left(\omega_{1}+\omega_{2}\right) / 2$. It is trivial to show in numerical examples that the bichromatic system is indeed equivalent to a monochromatic one with $\omega_{0}$ taken as the simple average of $\omega_{1}$ and $\omega_{2}$.

If we have different distributions for neutrinos and antineutrinos, we define the initial average frequencies by

$$
\left\langle\omega_{\nu}\right\rangle=\frac{\int_{0}^{\infty} \mathrm{d} \omega \omega P_{\omega}^{z}}{\int_{0}^{\infty} \mathrm{d} \omega P_{\omega}^{z}}
$$

and analogous for $\left\langle\omega_{\bar{\nu}}\right\rangle$. The equivalent monochromatic frequency is then $\omega_{0}=\frac{1}{2}\left(\left\langle\omega_{\nu}\right\rangle+\left\langle\omega_{\bar{\nu}}\right\rangle\right)$. The initial distribution $P_{\omega}^{z}$ can involve negative values if some part of the spectrum initially consists of $\nu_{x}$ and not $\nu_{e}$. Such spectral cross-overs occur, for example, if one assumes thermal fluxes with equal luminosities but different temperatures.

We have studied several numerical examples of quasi single-angle behavior and of multi-angle decoherence, taking different neutrino and antineutrino energy spectra, such as flat or thermal and with equal or different temperatures. We always found that the evolution of 
the global polarization vectors is almost identical to the equivalent monochromatic cases. We never observed that a broad energy spectrum caused a significant deviation from the monochromatic behavior at those radii that are relevant for decoherence.

Of course, a multi-energy system is qualitatively different from a monochromatic one in that the final energy distribution shows a "spectral split" [5, 6, 9, , 10, 11]. In a single-angle multi-energy system, this means the $\bar{\nu}_{e}$ spectrum is completely transformed to the $\bar{\nu}_{x}$ flavor, whereas only the high-energy part of the $\nu_{e}$ spectrum is transformed, the low-energy part remaining in (or rather returning to) the original flavor. The energy $E_{\text {split }}$ of this sharp transition is fixed by lepton-number conservation in the sense that the neutrino-neutrino interactions only catalyzes the transformation of $\nu_{e} \bar{\nu}_{e}$ pairs. For various examples we find results in full agreement with the previous literature [5, $\underline{6}, \underline{9}, \underline{10}, 11$ ].

For sufficiently large asymmetries $\epsilon$ where the multiangle system evolves in the quasi single-angle mode, there is no significant modification of the spectral split so that it is not worthwhile to show any examples. In the decoherent case, the final spectra naturally are very different, but we have not explored such cases systematically because multi-angle decoherence does not seem to be generic for realistic SN scenarios.

To illustrate the modifications caused by an energy spectrum in a different way from the previous literature, we show in Fig. 10 the side and top views of the location of neutrino and antineutrino polarization vectors on the unit sphere in analogy to Fig. 3 for our standard parameter values. In the left column we show the same monochromatic multi-angle case that we already showed in the left column of Fig. 3, with 500 modes. In addition we include the neutrinos (blue/dark gray) that here live on a sphere of radius $1+\epsilon=1.25$. The neutrinos form a spiral structure similar to the one of the antineutrinos, but in the final state this structure cannot move to the negative $\mathbf{B}$ directions because of lepton number conservation.

In the middle column we show a single-angle example with the same parameters, now using a box-like spectrum of oscillation frequencies where initially $\bar{P}_{\omega}^{z}=\left(2 \omega_{0}\right)^{-1}$ and $P_{\omega}^{z}=(1+\epsilon)\left(2 \omega_{0}\right)^{-1}$ for $0 \leq \omega \leq 2 \omega_{0}$ so that $\left\langle\omega_{\nu}\right\rangle=\left\langle\omega_{\bar{\nu}}\right\rangle=\omega_{0}$ and it is equivalent to the original monochromatic case. We now see that most of the antineutrinos have moved to the negative $\mathbf{B}$ direction as before, whereas the neutrinos populate both the positive and negative $\mathbf{B}$ direction, representing the spectral split. The lack of full adiabaticity prevents the split from being complete, leaving some polarization vectors not fully aligned or anti-aligned with $\mathbf{B}$. At large radii when the neutrino-neutrino interactions have died out, these modes precess with their different vacuum oscillation frequencies so that they are found on a spiral locus extending from the "south pole" to the "north pole" that gets wound up further at larger radii. Note that here we have used 1000 energy modes in order to obtain a visi- ble population occupying these non-adiabatic final states. Still, only very few red dots (antineutrinos) are visible, the vast majority being at the south pole. Likewise for the neutrinos (blue dots), the spiral is populated only by a small fraction of the 1000 modes. In other words, the evolution is nearly adiabatic.

Finally we combine a box-like energy spectrum and a multi-angle distribution (right panels). The antineutrinos all cluster around the negative $\mathbf{B}$ direction and fill the "southern polar cap" more or less uniformly because at late times modes with different energies precess with different frequencies. The neutrinos populate both the northern and southern polar caps, representing the spectral split. At intermediate latitudes we find coherent spiral structures. They correspond to modes with different angles but equal $\omega$ so that even at late times they do not dissolve by differential precession.

\section{Effective interaction strength}

Besides the asymmetry $\epsilon$ itself, the most uncertain model parameter is the effective neutrino-neutrino interaction strength $\mu$ as defined in Eq. (9). In Fig. 11] we show the demarcation lines between coherence and decoherence for both hierarchies in the $\mu$ - $\epsilon$-plane, keeping all other parameters at their standard values. The contours are constructed as described in Sec.IVB. The numerical contours are visually very well approximated by linear regressions of the form

$$
\begin{aligned}
\epsilon_{\mathrm{IH}} & \approx 0.225+0.027 \log _{10}\left(\frac{\mu}{10^{6} \mathrm{~km}^{-1}}\right) \\
\epsilon_{\mathrm{NH}} & \approx 0.172+0.087 \log _{10}\left(\frac{\mu}{10^{6} \mathrm{~km}^{-1}}\right) .
\end{aligned}
$$

For the normal hierarchy, the linear regression would intersect $\epsilon=0$ within the range of investigated $\mu$-values, but in reality turns over and saturates around $\epsilon=0.06$.

\section{E. Vacuum oscillation frequency}

The average vacuum oscillation frequency $\omega$ depends on the atmospheric $\Delta m^{2}$ that is quite well constrained, and a certain average of the neutrino energies. Our standard value is $\omega=0.3 \mathrm{~km}^{-1}$. If we increase this to $1 \mathrm{~km}^{-1}$, the $\epsilon$ - $\mu$-contour in Fig. 11] is essentially parallel-shifted to larger $\epsilon$ by about 0.035 (inverted hierarchy). This range of $\omega$ probably brackets the plausible possibilities so that the uncertainty of $\omega$ does not strongly influence the practical demarcation between the regimes.

The normal hierarchy is more sensitive to $\omega$. In Fig. 12 we show a contour for the coherence regime in the $\epsilon-\omega$ plane, assuming otherwise our standard parameter values. Changing $\omega$ from 0.3 to $1 \mathrm{~km}^{-1}$ increases the critical $\epsilon$ by almost 0.15 . 


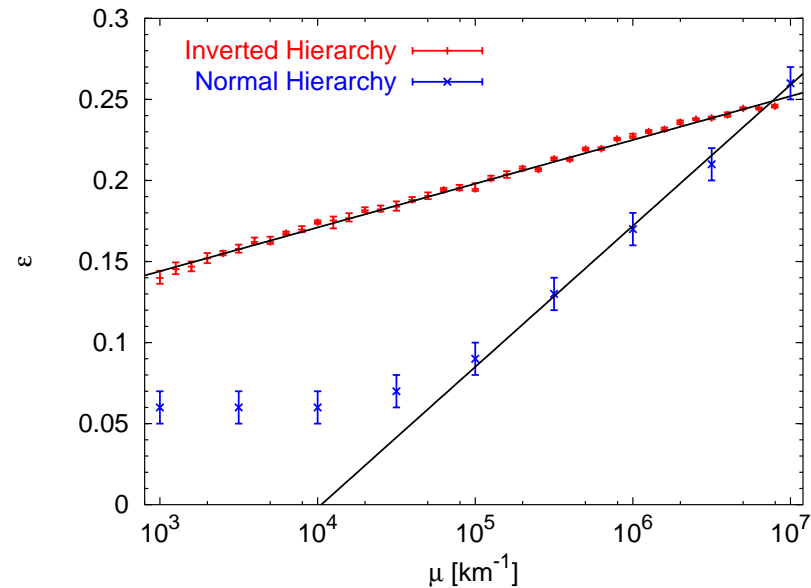

FIG. 11: Limiting $\epsilon$ for decoherence as a function of the effective neutrino-neutrino interaction strength $\mu$ for our standard parameters. The linear regressions are "visual fits" represented by Eq. (16).

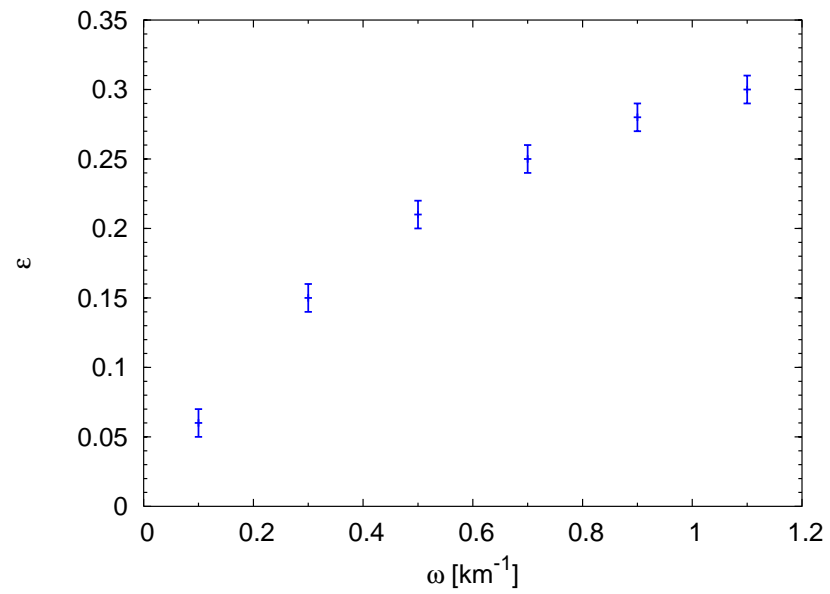

FIG. 12: Limiting $\epsilon$ for decoherence as a function of the vacuum oscillation frequency $\omega$ for our standard parameters and the normal hierarchy.

\section{CONCLUSIONS}

The nonlinear neutrino transformations that occur in the dense-neutrino region of a SN show numerous novel features. It was noted that multi-angle effects play an important role in that the neutrino-neutrino interaction depends on the relative angles of the various trajectories [5, 6]. At the same time it was numerically observed that for a typical example the behavior was unexpectedly quite similar to the single-angle case [5, 6]. On the other hand it was analytically shown that a gas of equal densities of neutrinos and anti-neutrinos has a pronounced angular instability and kinematical decoherence between different angular modes in flavor space is fast, representing a stable fixed point of the system [8].

We have here not attempted to develop further ana- lytical insights, but have taken a practical approach and explored numerically the range of parameters where different forms of behavior dominate in a realistic SN scenario.

To this end we have first clarified that "multi-angle effects" mean one of two clearly separated forms of behavior. The flavor content of the system can evolve in a quasi single-angle form. On the level of the polarization vectors this means that they fill only a restricted volume of the available phase space and maintain a coherent structure. On the other hand, nearly complete flavor equilibrium can arise where the available phase space is more or less uniformly filled.

For realistic assumptions about supernova and neutrino parameters, the switch between these modes of evolution is set by the degree of asymmetry between the neutrino and antineutrino fluxes. While this asymmetry is caused by the deleptonization flux, the crucial parameter $\epsilon$ is the asymmetry between $F\left(\nu_{e}\right)-F\left(\nu_{x}\right)$ and the corresponding antineutrino quantity as defined in Eq. (1) because for flavor oscillations the part of the density matrix that is proportional to $F\left(\nu_{e}\right)+F\left(\nu_{x}\right)$ drops out. While in a realistic SN on average $F\left(\nu_{e}\right)$ is about $15 \%$ larger than $F\left(\bar{\nu}_{e}\right)$, the asymmetry parameter as defined in Eq. (1) is typically much larger.

The critical value of $\epsilon$ that is enough to suppress decoherence depends on the type of neutrino mass hierarchy, the average energies, luminosities, and on the mixing angle. We have found that for $\epsilon \gtrsim 0.3$, decoherence is suppressed for the entire range of plausible parameters, but a value smaller than 0.1 may be enough, depending on the combination of other parameters.

We conclude that the quasi single-angle behavior may well be typical for realistic SN conditions, i.e., that the deleptonization flux is enough to suppress multi-angle decoherence. To substantiate this conclusion one should analyze the output of numerical simulations in terms of our model parameters. Besides the flavor-dependent luminosities and average energies, one needs the angular distribution of the neutrino radiation field at some radius where collisions are no longer important.

If our conclusion holds up in the light of realistic SN simulations, a practical understanding of the effect of selfinduced neutrino flavor transformations quickly comes into reach. In the normal mass hierarchy, nothing new would happen on a macroscopic scale. In the inverted hierarchy, the final effect would be a conversion of $\nu_{e} \bar{\nu}_{e}$ pairs and a split in the $\nu_{e}$ spectrum. These phenomena are only mildly affected by multi-angle effects as long as we are in the quasi single-angle regime.

If at late times the matter density profile contracts enough that an MSW effect occurs in the dense-neutrino region, the situation becomes more complicated as the neutrino-neutrino and ordinary matter effects interfere and produce a richer structure of spectral modifications [5, 6]. Even then, numerical simulations are much simpler if multi-angle decoherence is suppressed.

It is not obvious how $\epsilon$ evolves at late times. The delep- 
tonization of the core is probably faster than the cooling so that one may think that $\epsilon$ becomes smaller. On the other hand, the $\bar{\nu}_{e}$ can essentially only interact via neutral current reactions and their flux and energy distribution should, therefore, become very similar to the ones of $\nu_{x}$ and $\bar{\nu}_{x}$. Therefore, it is not obvious if at late times the initial flux difference $F_{\nu_{e}}-F_{\bar{\nu}_{e}}$ or $F_{\bar{\nu}_{e}}-F_{\bar{\nu}_{x}}$ decreases more quickly. We also note that there can be a crossover in the sense that at late times the flux hierarchy can become $F_{\nu_{x}}=F_{\bar{\nu}_{x}}>F_{\nu_{e}}>F_{\bar{\nu}_{e}}$ as in Ref. [13], meaning that we would have a pair excess flux of $\nu_{x} \bar{\nu}_{x}$ instead of a $\nu_{e} \bar{\nu}_{e}$ excess.

We have refrained from an interpretation of our numerical findings because we do not have developed a theory of kinematical decoherence for a system that is asymmetric between neutrinos and antineutrinos and where the effective interaction strength varies as a function of time (or here of radius). The absence of multi-angle decoherence seems to follow from a lack of time for it to develop. One can interpret our results such that a more adiabatic decrease of the neutrino-neutrino interaction strength requires a larger asymmetry to suppress decoherence. The different length scales of the problem seem to conspire such that the evolution is adiabatic in that sharp spectral splits develop, but not so adiabatic that kinematical decoherence would be typical. An analytic understanding of this conspiracy remains to be found.

Our results suggest that signatures of collective flavor transformations are not erased by multi-angle decoherence and will survive to the surface, modulated by the usual MSW flavor conversions [16]. The survival of observable signatures then also depends on the density fluctuations of the ordinary medium that can be a source of kinematical flavor decoherence [17, 18].

All authors in this field have relied on the simplifying assumption of either homogeneity or exact spherical symmetry to make the equations numerically tractable. The neutrino emission from a real SN is influenced by density and temperature fluctuations of the medium in the region where neutrinos decouple. Likewise, the neutrino fluxes emitted from the accretion tori of coalescing neutron stars, the likely engines of short gamma ray bursts, have fewer symmetries than assumed here. It remains to be investigated if systems with more general geometries behave qualitatively similar to the spherically symmetric case or if deviations from spherical symmetry can provide a new source of kinematical decoherence.

\section{Acknowledgments}

This work was partly supported by the Deutsche Forschungsgemeinschaft under the grant TR-27 "Neutrinos and Beyond", by The Cluster of Excellence for Fundamental Physics "Origin and Structure of the Universe" (Garching and Munich), by the European Union under the ILIAS project (contract No. RII3-CT-2004506222) and an RT Network (contract No. MRTN-CT-
2004-503369), and by the Spanish grants FPA2005-01269 (MEC) and ACOMP07-270 (Generalitat Valenciana). A. E. has been supported by a FPU grant from the Spanish Government. SP and RT were supported by MEC contracts (Ramón y Cajal and Juan de la Cierva, respectively).

\section{APPENDIX A: EQUATIONS OF MOTION}

\section{Temporal evolution}

A homogeneous ensemble of unmixed neutrinos is represented by the occupation numbers $f_{\mathbf{p}}=\left\langle a_{\mathbf{p}}^{\dagger} a_{\mathbf{p}}\right\rangle$ for each momentum mode $\mathbf{p}$, where $a_{\mathbf{p}}^{\dagger}$ and $a_{\mathbf{p}}$ are the relevant creation and annihilation operators and $\langle\ldots\rangle$ is the expectation value. A corresponding expression can be defined for the antineutrinos, $\bar{f}_{\mathbf{p}}=\left\langle\bar{a}_{\mathbf{p}}^{\dagger} \bar{a}_{\mathbf{p}}\right\rangle$, where overbarred quantities always refer to antiparticles. In a multiflavor system of mixed neutrinos, the occupation numbers are generalised to density matrices in flavor space [19, 20, 21]

$$
\left(\varrho_{\mathbf{p}}\right)_{i j}=\left\langle a_{i}^{\dagger} a_{j}\right\rangle_{\mathbf{p}} \quad \text { and } \quad\left(\varrho_{\mathbf{p}}\right)_{i j}=\left\langle\bar{a}_{j}^{\dagger} \bar{a}_{i}\right\rangle_{\mathbf{p}} .
$$

The reversed order of the flavor indices $i$ and $j$ in the right-hand side for antineutrinos assures that $\varrho_{\mathbf{p}}$ and $\bar{\varrho}_{\mathbf{p}}$ transform identically under a flavor transformation.

Flavor oscillations of an ensemble of neutrinos and antineutrinos are described by [19, 20, 21]

$$
\mathrm{i} \partial_{t} \varrho_{\mathbf{p}}=\left[\mathrm{H}_{\mathbf{p}}, \varrho_{\mathbf{p}}\right] \text { and } \mathrm{i} \partial_{t} \bar{\varrho}_{\mathbf{p}}=\left[\overline{\mathrm{H}}_{\mathbf{p}}, \bar{\varrho}_{\mathbf{p}}\right] \text {, }
$$

where $[\cdot, \cdot]$ is a commutator. The "Hamiltonian" for each mode is

$\mathrm{H}_{\mathbf{p}}=\Omega_{\mathbf{p}}+\lambda \mathrm{L}+\sqrt{2} G_{\mathrm{F}} \int \frac{\mathrm{d}^{3} \mathbf{q}}{(2 \pi)^{3}}\left(\varrho_{\mathbf{q}}-\bar{\varrho}_{\mathbf{q}}\right)\left(1-\mathbf{v}_{\mathbf{q}} \cdot \mathbf{v}_{\mathbf{p}}\right)$,

where $G_{\mathrm{F}}$ is the Fermi constant. The matrix of vacuum oscillation frequencies for relativistic neutrinos is in the mass basis $\Omega_{\mathbf{p}}=\operatorname{diag}\left(m_{1}^{2}, m_{2}^{2}, m_{3}^{2}\right) / 2 p$ with $p=|\mathbf{p}|$. The matter effect is represented by $\lambda=\sqrt{2} G_{\mathrm{F}}\left(n_{e^{-}}-n_{e^{+}}\right)$ and $\mathrm{L}=\operatorname{diag}(1,0,0)$, given here in the weak interaction basis. We ignore the possible presence of other chargedlepton flavors. The Hamiltonian for antineutrinos $\bar{H}_{p}$ is the same with $\Omega_{\mathbf{p}} \rightarrow-\Omega_{\mathbf{p}}$, i.e., in vacuum antineutrinos oscillate "the other way round."

The factor $\left(1-\mathbf{v}_{\mathbf{q}} \cdot \mathbf{v}_{\mathbf{p}}\right)=\left(1-\cos \theta_{\mathbf{p q}}\right)$ represents the current-current nature of the weak interaction where $\mathbf{v}_{\mathbf{p}}=\mathbf{p} / p$ is the velocity. The angular term averages to zero if the gas is isotropic. We ignore a possible net flux of charged leptons lest the ordinary matter effect also involves an angular factor.

If the system is axially symmetric relative to some direction, the angular factor simplifies after an azimuthal integration to [5, 8 ]

$$
\left(1-\mathbf{v}_{\mathbf{q}} \cdot \mathbf{v}_{\mathbf{p}}\right) \rightarrow\left(1-v_{\mathbf{q}} v_{\mathbf{p}}\right),
$$

where the velocities are along the symmetry axis. 


\section{Spatial evolution in spherical symmetry}

Instead of a homogeneous system that evolves in time we consider a stationary system that evolves in space. The occupation numbers become Wigner functions, which depend both on spatial coordinates and on momenta, but there is no conceptual problem as long as we consider spatial variations that are slow on the scale of the inverse neutrino momenta.

Since multi-angle effects are at the focus of our problem, we cannot reduce the equations to plane waves moving in the same direction. Motivated by the SN application, however, we can take advantage of global spherical symmetry, implying that the ensemble is represented by matrices that depend on a radial coordinate $r$, the zenith angle relative to the radial direction, and the energy $E$ which in the relativistic limit is identical with $p=|\mathbf{p}|$.

We ignore gravitational deflection near the SN core and assume that neutrinos move on straight lines after being launched at a radius $R$ that we call the neutrino sphere. Consider a neutrino that was launched at an angle $\vartheta_{R}$ relative to the radial direction. Its radial velocity is

$$
v_{R}=\cos \vartheta_{R}
$$

At $r>R$ the trajectory's angle relative to the radial direction is implied by simple geometry to be [5] (see e.g. their Fig. 1)

$$
R \sin \vartheta_{R}=r \sin \vartheta_{r}
$$

Therefore, the radial velocity at $r$ is

$$
v_{u, r}=\cos \vartheta_{r}=\sqrt{1-\frac{R^{2}}{r^{2}} u}
$$

where we have introduced

$$
u=1-v_{R}^{2}=\sin ^{2} \vartheta_{R} .
$$

It is convenient to label the angular modes with $u$. The physical zenith angles change with distance so that the equations would be more complicated.

The density matrices $\varrho_{p, u, r}$ are not especially useful to describe a spherically symmetric system because they vary with $r$ even in the absence of oscillations. (Note that we often write the dependence of a quantity on a variable as an subscript.) A quantity that is conserved in the absence of oscillations is the total flux matrix

$$
\mathrm{J}_{r}=\frac{r^{2}}{R^{2}} \int \frac{\mathrm{d}^{3} \mathbf{p}}{(2 \pi)^{3}} \varrho_{\mathbf{p}, r} v_{\mathbf{p}, r} .
$$

To express the integral in co-moving variables we observe that $\mathrm{d}^{3} \mathbf{p}$ in spherical coordinates is $p^{2} \mathrm{~d} p \mathrm{~d} \varphi \mathrm{d} \cos \vartheta_{r}$ and that Eq. (A7) implies

$$
\left|\frac{\mathrm{d} \cos \vartheta_{r}}{\mathrm{~d} u}\right|=\frac{1}{2 v_{u, r}} \frac{R^{2}}{r^{2}} .
$$

Therefore, we finally define the differential flux matrices

$$
\mathrm{J}_{p, u, r}=\frac{p^{2} \varrho_{p, u, r}}{2(2 \pi)^{2}}
$$

where we have used $\int \mathrm{d} \varphi=2 \pi$ for axial symmetry. The normalization is

$$
\mathrm{J}_{r}=\int_{0}^{1} \mathrm{~d} u \int_{0}^{\infty} \mathrm{d} p \mathrm{~J}_{p, u, r}
$$

In the absence of oscillations the total and differential fluxes are conserved, $\partial_{r} \mathrm{~J}_{r}=0$ and $\partial_{r} \mathrm{~J}_{p, u, r}=0$.

To include oscillations, we note that the radial velocity along a neutrino trajectory is $v_{u, r}=\mathrm{d} r_{u} / \mathrm{d} t=\cos \vartheta_{u, r}$. Therefore, if we wish to express the temporal evolution of the neutrino density matrix along its trajectory in terms of an evolution expressed in terms of the radial coordinate $r$, we substitute $\partial_{t} \rightarrow v_{u, r} \partial_{r}$ in Eq. (A2) so that

$$
\mathrm{i} \partial_{r} \mathrm{~J}_{p, u, r}=\frac{\left[\mathrm{H}_{p, u, r}, \mathrm{~J}_{p, u, r}\right]}{v_{u, r}},
$$

and analogous for antineutrinos. In other words, we project the evolution along a given trajectory to an evolution along the radial direction. For vacuum oscillations this has the effect of "compressing" the oscillation pattern for non-radial modes, i.e., even for monochromatic neutrinos, the effective vacuum oscillation frequency depends on both $r$ and $u$.

The vacuum-oscillation and ordinary-matter contributions to $\mathrm{H}_{p, u, r}$ were given in Eq. (A3), whereas the selfterm must be made explicit. To this end we introduce the matrix of number densities

$$
\mathrm{N}_{p, u, r}=v_{u, r}^{-1} \mathrm{~J}_{p, u, r}
$$

and its integral as

$$
\mathrm{N}_{r}=\int_{0}^{1} \mathrm{~d} u \int_{0}^{\infty} \mathrm{d} p \mathrm{~N}_{p, u, r}=\int_{0}^{\infty} \mathrm{d} u \int_{0}^{\infty} \mathrm{d} p \frac{\mathrm{J}_{p, u, r}}{v_{u, r}}
$$

Collecting all terms and taking advantage of Eq. (A4) for axial symmetry, we find

$$
\begin{aligned}
& \mathrm{i} \partial_{r} \mathrm{~J}_{p, u, r}=+\left[\Omega_{p}, \mathrm{~N}_{p, u, r}\right]+\lambda_{r}\left[\mathrm{~L}, \mathrm{~N}_{p, u, r}\right]+\sqrt{2} G_{\mathrm{F}} \frac{R^{2}}{r^{2}}\left(\left[\mathrm{~N}_{r}-\overline{\mathrm{N}}_{r}, \mathrm{~N}_{p, u, r}\right]-\left[\mathrm{J}_{r}-\overline{\mathrm{J}}_{r}, \mathrm{~J}_{p, u, r}\right]\right), \\
& \mathrm{i} \partial_{r} \overline{\mathrm{J}}_{p, u, r}=-\left[\Omega_{p}, \overline{\mathrm{N}}_{p, u, r}\right]+\lambda_{r}\left[\mathrm{~L}, \overline{\mathrm{N}}_{p, u, r}\right]+\sqrt{2} G_{\mathrm{F}} \frac{R^{2}}{r^{2}}\left(\left[\mathrm{~N}_{r}-\overline{\mathrm{N}}_{r}, \overline{\mathrm{N}}_{p, u, r}\right]-\left[\mathrm{J}_{r}-\overline{\mathrm{J}}_{r}, \overline{\mathrm{J}}_{p, u, r}\right]\right),
\end{aligned}
$$


where the electron density's radial variation is included in $\lambda_{r}$.

\section{Angular emission characteristics}

In a numerical simulation we need to specify the fluxes at the neutrino sphere $r=R$. For our usual multiangle simulations we assume that the neutrino radiation field is "half isotropic" directly above the neutrino sphere, i.e., that all outward-moving angular modes are equally occupied as behooves a thermal radiation field. Therefore, the occupation numbers are distributed as $\mathrm{d} n / \mathrm{d} \cos \vartheta_{R}=$ const., implying that the radial fluxes are distributed as $\mathrm{d} j / \mathrm{d} \cos \vartheta_{R}=v_{R} \mathrm{~d} n / \mathrm{d} \cos \vartheta_{R} \propto \cos \vartheta_{R}$ because $v_{R}=\cos \vartheta_{R}$. Expressed in the angular variable $u$ this implies $\mathrm{d} j / \mathrm{d} u=$ const. because of Eq. (A10). In other words, a blackbody radiation field at the neutrino sphere implies that

$$
\mathrm{J}_{u}=\text { const. }
$$

in the interval $0 \leq u \leq 1$.

To avoid multi-angle effects one may sometimes wish to use a single angular bin. To represent a uniform $J_{u}$ distribution, the natural choice is $u=1 / 2$, corresponding to a launch angle $\vartheta_{R}=45^{\circ}$. Our numerical single-angle examples always correspond to this choice in an otherwise unchanged numerical code.

In this case the radial velocity of all neutrinos as a function of radius is

$$
v_{r}=\sqrt{1-\frac{R^{2}}{2 r^{2}}} .
$$

For a monochromatic spectrum, the remaining flavor matrices are simply the total $\mathrm{J}_{r}$ (corresponding to the single $u=1 / 2$ ) and $\mathrm{N}_{r}=\mathrm{J}_{r} / v_{r}$. Ignoring the trivial ordinary matter term, the equations of motion are

$$
\mathrm{i} \partial_{r} \mathrm{~J}_{r}=\frac{\left[\Omega, \mathrm{J}_{r}\right]}{v_{r}}+\sqrt{2} G_{\mathrm{F}} \frac{R^{2}}{r^{2}}\left(\frac{1}{v_{r}^{2}}-1\right)\left[\mathrm{J}_{r}-\overline{\mathrm{J}}_{r}, \mathrm{~J}_{r}\right]
$$

and analogous for the antineutrinos. The coefficient of the neutrino-neutrino term is explicitly

$$
\sqrt{2} G_{\mathrm{F}} \frac{R^{4}}{r^{4}} \frac{1}{2-R^{2} / r^{2}} .
$$

At the neutrino sphere it is equal to $\sqrt{2} G_{\mathrm{F}}$, whereas at large distances it is $\left(\sqrt{2} G_{\mathrm{F}} / 2\right) R^{4} / r^{4}$. As observed in the previous literature, the neutrino-neutrino term dies out at large distances as $r^{-4}$.

One can define a "single-angle case" somewhat differently. Assuming all angular modes evolve coherently, we can integrate the equations of motion over $\int_{0}^{1} \mathrm{~d} u$ and study the evolution of the quantities $J_{p, r}=\int_{0}^{1} \mathrm{~d} u J_{p, u, r}$. To write the equations in a compact form we introduce the notation

$$
\frac{1}{v_{r}^{*}} \equiv \frac{1}{\mathrm{~J}_{r}} \int_{0}^{\infty} \mathrm{d} p \int_{0}^{1} \mathrm{~d} u \frac{\mathrm{J}_{p, u, r}}{v_{u, r}} .
$$

The full equation of motion Eq. (A16) for neutrinos becomes

$$
\begin{aligned}
\mathrm{i} \partial_{r} \mathrm{~J}_{p, r}= & \frac{\left[\Omega_{p}, \mathrm{~J}_{p, r}\right]}{v_{r}^{*}}+\lambda_{r} \frac{\left[\mathrm{L}, \mathrm{J}_{p, r}\right]}{v_{r}^{*}} \\
& +\sqrt{2} G_{\mathrm{F}} \frac{R^{2}}{r^{2}}\left(\frac{1}{\left(v_{r}^{*}\right)^{2}}-1\right)\left[\mathrm{J}_{r}-\overline{\mathrm{J}}_{r}, \mathrm{~J}_{p, r}\right]
\end{aligned}
$$

and analogous for antineutrinos with $\Omega_{p} \rightarrow-\Omega_{p}$.

At large distances we have $1 / v_{r}^{*}=1+\frac{1}{2}(R / r)^{2}\langle u\rangle$ where $\langle u\rangle$ is the average of $u$ at emission. For the vacuum and matter terms, we only need the leading terms so that we recover the familiar plane-wave form of the equations of motion. The coefficient of the neutrino-neutrino term, on the other hand, becomes

$$
\sqrt{2} G_{\mathrm{F}} \frac{R^{4}}{r^{4}}\langle u\rangle
$$

Both for half-isotropic emission and for our single-angle case we have $\langle u\rangle=\frac{1}{2}$, in agreement with Eq. (A20).
[1] S. Pastor and G. Raffelt, Phys. Rev. Lett. 89, 191101 (2002) astro-ph/0207281.

[2] R.F. Sawyer, Phys. Rev. D 72, 045003 (2005) hep-ph/0503013.

[3] G.M. Fuller and Y.Z. Qian, Phys. Rev. D 73, 023004 (2006) astro-ph/0505240.

[4] H. Duan, G.M. Fuller and Y.Z. Qian, Phys. Rev. D 74, 123004 (2006) astro-ph/0511275.

[5] H. Duan, G.M. Fuller, J. Carlson and Y.Z. Qian, Phys. Rev. D 74, 105014 (2006) astro-ph/0606616.

[6] H. Duan, G.M. Fuller, J. Carlson and Y.Z. Qian, Phys. Rev. Lett. 97, 241101 (2006) astro-ph/0608050.

[7] S. Hannestad, G.G. Raffelt, G. Sigl and Y.Y.Y. Wong,
Phys. Rev. D 74, 105010 (2006) astro-ph/0608695.

[8] G.G. Raffelt and G. Sigl, Phys. Rev. D 75, 083002 (2007) hep-ph/0701182.

[9] H. Duan, G.M. Fuller, J. Carlson and Y.Z. Qian, Phys. Rev. D 75, 125005 (2007) astro-ph/0703776.

[10] G.G. Raffelt and A.Yu. Smirnov, Phys. Rev. D 76, 081301 (2007) [hep-ph/0705.1830].

[11] G.L. Fogli, E. Lisi, A. Marrone and A. Mirizzi, JCAP 0712, 010 (2007) arXiv:0707.1998.

[12] M. Kachelrießet al, Phys. Rev. D 71, 063003 (2005) astro-ph/0412082.

[13] G.G. Raffelt et al, Proc. of the 4th Workshop on Neutrino Oscillations and their Origin: NooN 
2003 (10-14 February 2003, Kanazawa, Japan), eds. Y. Suzuki, M. Nakahata, Y. Itow, M. Shiozawa and Y. Obayashi (World Scientific, Singapore, 2004), pp. 380387 astro-ph/0303226.

[14] A. Arcones, H.-T. Janka and L. Scheck, Astron. Astrophys. 467, 1227 (2007) astro-ph/0612582.

[15] V.A. Kostelecký and S. Samuel, Phys. Rev. D 52, 621 (1995) hep-ph/9506262.

[16] A.S. Dighe and A.Yu. Smirnov, Phys. Rev. D 62, 033007 (2000) hep-ph/9907423.
[17] G.L. Fogli, E. Lisi, A. Mirizzi and D. Montanino, JCAP 0606, 012 (2006) hep-ph/0603033.

[18] A. Friedland and A. Gruzinov, preprint astro-ph/0607244

[19] A.D. Dolgov, Yad. Fiz. 33, 1309 (1981) [Sov. J. Nucl. Phys. 33, 700 (1981)].

[20] G. Sigl and G.G. Raffelt, Nucl. Phys. B 406, 423 (1993).

[21] B.H.J. McKellar and M.J. Thomson, Phys. Rev. D 49, 2710 (1994). 